\title{
Propuesta de indicadores ambientales para un turismo sostenible en el desierto de la Tatacoa, Huila, Colombia*
}

\author{
Recibido: 19 de abril de 2020 - Aprobado: 8 de junio de 2020 \\ https://doi.org/10.22395/seec.v23n54a12 \\ José Jardani Giraldo Uribe ${ }^{* *}$ \\ María del Pilar Sánchez Muñoz ** \\ Héctor Hugo Ruiz Agudelo****
}

\section{RESUMEN}

Al considerar el auge que actualmente experimenta el turismo en desiertos y sus efectos, se presenta una propuesta de indicadores para conocer la valoración ambiental en 2019, expresada a través de un índice sintético denominado índice ambiental para el turismo sostenible (IATS) en el desierto de la Tatacoa. La metodología consiste en seleccionar un conjunto de indicadores que toman como referencia la propuesta de indicadores para el turismo sostenible de la Organización Mundial del Turismo, algunos criterios de selección de indicadores y las características específicas del territorio. El resultado es una propuesta que se compone de veintiocho indicadores, de los cuales ocho fueron calculados para la construcción del IATS, el cual permite valorar el estado ambiental del destino turístico como bueno.

\section{PALABRAS CLAVE}

Desierto; turismo en desiertos; turismo sostenible; indicadores ambientales.

\section{CLASIFICACIÓN JEL}

L83, Q51, Q01

\section{CONTENIDO}

Introducción; 1. Turismo sostenible e indicadores en entornos desérticos; 2. Metodología; 3. Resultados; 4. Conclusiones; Bibliografía.

Este artículo de investigación es resultado del proyecto n. 3015 Propuesta de indicadores ambientales para un turismo sostenible en el desierto de la Tatacoa. Esta producción académica contó con financiación en tiempo y recursos financieros de la Universidad Surcolombiana, según Acta n. 021 del 29 de noviembre de 2018.

*. Economista, Universidad Libre, Pereira, Colombia. Magíster en Administración Económica y Financiera, Universidad Tecnológica de Pereira, Pereira, Colombia. Doctor en Desarrollo Sostenible, Universidad de Manizales, Caldas, Colombia. Docente de tiempo completo de la Universidad Surcolombiana, Huila, Colombia. Investigador del Grupo Iguaque. Correo electrónico: jose.giraldo@usco.edu.co. Orcid: https://orcid.org/0000-0001-9198-6300

-.. Economista, Universidad Católica de Colombia, Bogotá, Colombia. Magíster en Economía, Universidad Nacional de Colombia, Bogotá, Colombia. Doctora en Desarrollo Sostenible, Universidad de Manizales, Caldas, Colombia. Docente Facultad de Economía de la Universidad del Rosario, Bogotá, Colombia. Investigadora del Grupo de Investigación Economía y Desarrollo Humano de la Universidad de La Salle, Bogotá, Colombia. Asesora metodológica y de desarrollo sostenible, Universidad Surcolombiana, Neiva, Colombia. Correo electrónico: mariad.sanchez@urosario.edu. Orcid: http://orcid.org/0000-0002-9325-9091

.... Administrador de Empresas. Especialista en Alta Gerencia. Docente de tiempo completo de la Universidad Surcolombiana, Huila, Colombia. Investigador del Grupo Iguaque. Correo electrónico: hector.ruiz@usco.edu.co. Orcid: https://orcid.org/0000-0002-7790-6949 


\title{
Environmental indicators for sustainable tourism proposal. Case of the la Tatacoa desert, Huila, Colombia
}

\begin{abstract}
By considering the boom in which deserts tourism currently is and its effects, here we present an indicators proposal to know the environmental values of 2019, expressed through an index known as sustainable tourism index (STI) in the Tatacoa desert. The methodology employed consists in selecting a group of indicators that take as a reference the sustainable tourism index of the World Tourism Organization, some of the criteria for selecting indicators and the specific features of the territory. The result is a proposal composed by 28th indicators from which eight were calculated for the construction of the STI, which allows the assessment of the environmental status of the tourist site as good.
\end{abstract}

\section{KEY WORDS}

Desert; Desert tourism; sustainable tourism; environmental indicators

\section{JEL CLASSIFICATION}

L83, Q51, Q01

\section{CONTENT}

Introduction; 1. Sustainable tourism and indicators in desert environments; 2. Methodology; 3. Results; 4. Conclusions; Bibliography.

\section{Proposta de indicadores ambientais para o turismo sustentável no deserto de la Tatacoa}

\section{RESUMO}

Ao considerar o auge atual do turismo em desertos e seus efeitos, é apresentada uma proposta de indicadores para conhecer a avaliação ambiental em 2019, expressa pelo índice sintético denominado "índice ambiental para o turismo sustentável" (IATS) no deserto da Tatacoa. A metodologia consiste em selecionar um conjunto de indicadores que tomam como referência a proposta de indicadores para o turismo sustentável da Organização Mundial do Turismo, alguns critérios de seleção de indicadores e das características específicas do território. O resultado é uma proposta composta de 28 indicadores, dos quais 8 foram calculados para a construção do IATS, o que permite avaliar o estado ambiental do destino turístico como bom.

\section{PALAVRAS CHAVE}

Deserto; turismo em desertos; turismo sustentável; indicadores ambientais

\section{JEL RATING}

L83, Q51, Q01

\section{CONTEÚDO}

Introdução; 1. Turismo sustentável e indicadores em ambientes desérticos; 2. Metodologia; 3. Resultados; 4. Conclusões; Bibliografia. 


\section{INTRODUCCIÓN}

En el siglo XXI el auge del turismo ha aumentado la demanda de sitios exóticos en el mundo, entre estos sitios se encuentran los desiertos. Este fenómeno ha generado una presión sobre dichos ecosistemas que puede impactar de forma negativa las dimensiones ambientales. El desierto de la Tatacoa, en Colombia, con un promedio de treinta y cuatro mil turistas al mes entre octubre de 2018 y octubre de 2019 (Hernández, 2019), no es ajeno a esta situación.

Al tener en cuenta que el territorio en sí es la base de la actividad turística, la dimensión medio ambiental es esencial para la cuantificación de la sostenibilidad. Por lo tanto, se presenta una propuesta de indicadores ambientales que permite conocer la valoración en 2019, expresada a través de un índice sintético ambiental denominado IATS (Índice Ambiental para el Turismo Sostenible), como insumo para la toma de decisiones en torno a la planificación y gestión del desierto de la Tatacoa como sitio turístico sostenible. Este índice es un punto de partida que debe ser complementado y actualizado con regularidad. Para este fin, se toma como marco de referencia la propuesta de indicadores para el turismo sostenible de la OMT (2005), los criterios de selección de indicadores de Medrano y Lardiés (2014) y las características específicas del desierto de la Tatacoa. Se cuenta con información procedente de la Alcaldía de Villavieja, la Corporación Autónoma del Alto Magdalena (CAM), Empresas Públicas de Villavieja (EPV SAS ESP.) y Planeación Nacional.

La propuesta cuenta con nueve dimensiones y veintiocho indicadores, de los cuales se cuenta con información para cuatro dimensiones que corresponden a ocho indicadores, constituyéndose este aspecto en la principal limitación de la investigación al imposibilitarse la cuantificación de veinte indicadores. El resultado principal de este estudio es la construcción del índice sintético ambiental IATS, el cual permite valorar el estado ambiental del destino turístico como bueno. No obstante, las limitaciones antes señaladas hacen que este resultado se deba interpretar con precaución; y convoca a los diferentes actores y tomadores de decisión a que sean conscientes de producir las estadísticas necesarias que sirvan de insumo para el adecuado seguimiento y evaluación de la planificación y gestión ambiental del desierto de la Tatacoa.

El documento consta de seis partes al incluir la presente introducción. En la segunda, se ofrecen algunos referentes sobre el turismo sostenible y los indicadores en entornos desérticos, de los cuales existen pocos estudios. En la tercera parte se describe la metodología utilizada para la propuesta y se explica la construcción del índice IATS. En la cuarta parte se presentan los resultados del estudio analizados para cinco dimensiones. Finalmente, se encuentran las conclusiones de la investigación y los referentes bibliográficos. 


\section{TURISMO SOSTENIBLE E INDICADORES EN ENTORNOS DESÉRTICOS}

Según la OMT (2020), desde el año 2009 el turismo ha presentado un notable crecimiento. En el año 2019 hubo mil quinientos millones de turistas internacionales, un 4 \% más que en 2018 y suma diez años de crecimiento continuo. De esta manera, se ha convertido en una actividad económica de gran peso a nivel de comercio internacional. Sin embargo, este aumento en el número de turistas genera efectos económicos, sociales en las comunidades locales y efectos ambientales negativos de gran repercusión, tales como la gestión inadecuada del agua, el aumento en la generación de los residuos sólidos, la planificación espontánea, la pérdida de los atractivos turísticos, la destrucción del producto turístico y la masificación acelerada. En este contexto, cobra importancia el término turismo sostenible.

De acuerdo con Mamani (2017):

El turismo sostenible tiene plenamente en cuenta las repercusiones actuales y futuras, económicas, sociales y medioambientales para satisfacer las necesidades de los visitantes, de la industria, del entorno y de las comunidades anfitrionas [además] debe reportar también un alto grado de satisfacción a los turistas y representar para ellos una experiencia significativa, que los haga más conscientes de los problemas de la sostenibilidad y fomente en ellos unas prácticas turísticas sostenibles. (párr. 1 y 5)

El año 2017 fue designado por las Naciones Unidas como el Año Internacional del Turismo Sostenible para el Desarrollo. De esta manera, se impulsa el concepto para que toda la actividad turística continué el proceso hacia el turismo sostenible. En este contexto, las regiones desérticas del mundo también están siendo sometidas a la llegada masiva de turistas, este es el caso de Marruecos con siete millones de turistas en 2018 y Kuwait que aspira a tener en el año 2020 cerca de veinte millones de turistas, como países más representativos.

Los desiertos ocupan cerca de una cuarta parte del territorio del mundo, entre ellos se caracterizan las principales áreas desérticas del mundo, como el desierto del Sahara y el Gran Desierto australiano, el desierto de Atacama en Chile y las zonas áridas de China. En Colombia existen cuatro desiertos. El primero se ubica en la Guajira; el segundo, la Candelaria, en el departamento de Cundinamarca; el tercero, en la zona árida de Antioquia, y el cuarto, el desierto de la Tatacoa, en el municipio de Villavieja (Departamento del Huila, Colombia).

En términos generales, los tipos de desiertos que existen son: i) desiertos continentales de latitudes medias, ii) desiertos de América del Norte, iii) desiertos costeros, iv) desiertos australianos, v) desiertos fríos subpolares y de montaña, y vi) desiertos tropicales y subtropicales. Debido al gran crecimiento 
del turismo, estos territorios desérticos, que antes habían permanecido relativamente aislados, están siendo visitados dada la demanda de sitios exóticos y el mejoramiento de los medios de transporte, lo que afecta dichos ecosistemas de diferentes formas. Estos efectos se manifiestan en los aspectos sociales (al chocar diferencias culturales y formas de ver el mundo), los efectos ambientales (en el agua, el aire, la tierra, la fauna y flora) y los efectos económicos.

Así, para el desarrollo adecuado y sostenible de la actividad turística, se hace indispensable una planeación y gestión estratégicas. Por lo anterior, es importante contar con indicadores de turismo sostenible que tenga en cuenta los desiertos, de tal manera que permitan medir y evaluar el tránsito de estos ecosistemas al turismo sostenible. Sin embargo, la concepción de mantener un equilibrio entre lo social, lo económico y ambiental debe ser complementada con un concepto de regeneración que permita mantener y aumentar el recurso natural en pos de la generación actual y la futura, en la línea del desarrollo sostenible iniciada desde el Informe Brundtland.

Según la OECD (2005), los indicadores ambientales miden un valor derivado de parámetros, que describen el estado de un fenómeno, el medio ambiente o un área, con un significado que va más allá del directamente vinculado con el valor del parámetro en sí mismo. Siguiendo a Cordero (2017) existen, en términos generales, dos modelos para evaluar el comportamiento ambiental. El modelo PER (presión, estado, resultado) de la OCDE y el modelo DPSIR (fuerza directriz, presión, estado, impacto y respuesta). El esquema PER está basado en una lógica de causalidad: las actividades humanas ejercen presiones sobre el ambiente y cambian la calidad y cantidad de los recursos naturales (estado). Asimismo, la sociedad responde a estos cambios a través de políticas ambientales, económicas y sectoriales (respuestas). Este modelo parte de cuestionamientos simples:

- ¿Qué está afectando al ecosistema desértico?

- ¿Qué está pasando con el estado del ecosistema desértico?

- ¿Qué acciones se están haciendo acerca del ecosistema desértico?

El modelo DPSIR es una derivación del modelo PER. Los indicadores correspondientes se refieren a las actividades humanas que generan las presiones (en otros modelos, presiones indirectas), los indicadores de estado se restringen a la situación del recurso ambiental y los de impacto muestran los efectos en la salud humana o los ecosistemas. Este modelo es impulsado por la Unión Europea. Con relación a los indicadores de turismo sostenible en desiertos, se identificaron cinco artículos: dos tratan sobre indicadores sociales, económicos y ambientales aplicados al territorio (Khalaf et al., 2019; Woyo y Amadhila, 2018); uno se aplica a 
las empresas turísticas (Del Bon et al., 2019); otro a degradación de tierras (Sallam et al., 2018); finalmente, solo uno estudia el uso de indicadores ambientales (Car et al., 2018). Este aspecto evidencia la poca producción científica alrededor de medir el impacto de la actividad turística sostenible en el territorio desértico.

\section{METODOLOGÍA}

Se realiza una investigación que busca la generación de conocimiento con aplicación directa a la problemática turística del desierto de la Tatacoa. Se toma como marco de referencia la propuesta de indicadores para el turismo sostenible de la OMT $(2005)^{1}$, los criterios de selección de indicadores de Medrano y Lardiés (2014) y las características específicas del desierto de la Tatacoa como destino turístico.

Del total del sistema de indicadores propuesto por la OMT (2005) para los tres ámbitos del desarrollo sostenible (económico, social y ambiental), se seleccionan los que están relacionados de forma directa con lo ambiental y con las características propias del turismo en desiertos. Esta revisión arroja un total de veintiocho indicadores relativos a las dimensiones: planificación territorial, paisaje, masificación y agua, planteadas por Giraldo (2018). Asimismo, se incluyen: residuos sólidos, calidad del aire, especies, ruido y energía.

Una vez seleccionados los veintiocho indicadores ambientales, estos se depuran de acuerdo con los criterios expuestos por Medrano y Lardiés (2014) para obtener, tanto la línea base del desierto de la Tatacoa que permita hacer seguimiento a través del tiempo, como la determinación de un índice ambiental sintético para 2019, denominado IATS (índice ambiental para el turismo sostenible). Los criterios de selección empleados son:

A. Que sean relevantes para conocer el medio ambiente del desierto de la Tatacoa desde la perspectiva turística.

B. Que los datos para su construcción estén disponibles y sean fáciles de obtener de las diferentes entidades encargadas.

\footnotetext{
En octubre de 2018, el grupo de trabajo sobre medición del turismo sostenible de la Organización Mundial del Turismo (OMT) se reunió en Madrid para dar impulso a la iniciativa de adoptar nuevos lineamientos para la medición del turismo sostenible, como tercera norma internacional sobre estadísticas de turismo. Sin embargo, a marzo de 2020 no ha sido publicado un nuevo documento oficial sobre el tema. La guía de 2005 describe trece temas relacionados con el desarrollo sostenible para los destinos turísticos que abarcan desde el bienestar de las comunidades receptoras, la conservación del patrimonio cultural, la participación comunitaria en el turismo, la satisfacción de los turistas, la salud y la seguridad, hasta el aprovechamiento de los beneficios económicos del turismo, la protección de los recursos naturales de valor, la gestión de recursos naturales escasos, la limitación del impacto ambiental del turismo, el control de las actividades turísticas, la ordenación y el control del lugar de destino, el diseño de productos y servicios, y la sostenibilidad de las operaciones y servicios turísticos.
} 
C. Que puedan ser actualizados con regularidad.

D. Que sean de fácil interpretación.

A partir de la depuración, se encuentra que veinte indicadores no poseen datos para su construcción y, por lo tanto, no han sido actualizados con regularidad, mientras que ocho de ellos sí cumplen los cuatro criterios establecidos por Medrano y Lardiés (2014). La tabla 1 presenta los indicadores que no cumplen los criterios B y C, pero son importantes para el monitoreo del turismo sostenible en el desierto de la Tatacoa.

Tabla 1. Indicadores por dimensión que no cumplen todos los criterios B y C

\begin{tabular}{|c|c|}
\hline \multicolumn{2}{|r|}{ Residuos sólidos } \\
\hline 1 & Imagen de limpieza del destino \\
\hline 2 & Residuos atribuibles (mes o estación) al turismo \\
\hline \multicolumn{2}{|r|}{ Calidad del aire } \\
\hline 3 & Percepción de la calidad del aire por parte de la comunidad local \\
\hline \multicolumn{2}{|r|}{ Paisaje } \\
\hline 4 & Número de elementos que emiten luz directa \\
\hline \multicolumn{2}{|r|}{ Especies } \\
\hline 5 & Salud de la población de las principales especies \\
\hline 6 & Índice de éxito reproductivo de las especies seleccionadas \\
\hline 7 & Costo de protección/repoblación \\
\hline 8 & Aportación del turismo a la protección y la repoblación \\
\hline \multicolumn{2}{|r|}{ Agua } \\
\hline 9 & Nivel de contaminación del agua del desierto (coliformes fecales y campilobacter) \\
\hline 10 & Nivel de contaminación del agua del desierto (metales pesados) \\
\hline 11 & Turbidez del agua (análisis sencillos) \\
\hline 12 & Percepción de la calidad del agua según los turistas \\
\hline 13 & Porcentaje de las aguas residuales tratadas \\
\hline \multicolumn{2}{|r|}{ Ruido } \\
\hline 14 & Niveles de ruido registrados en el DT medidos en decibeles (hora) \\
\hline 15 & Percepción del ruido sobre la comunidad local \\
\hline \multicolumn{2}{|r|}{ Masificación } \\
\hline 16 & Porcentaje de turistas y residentes locales que creen que el destino está masificado \\
\hline
\end{tabular}


José Jardani Giraldo Uribe, María del Pilar Sánchez Muñoz y Héctor Hugo Ruiz Agudelo

\section{Energía}

\begin{tabular}{lc}
\hline 17 & Consumo percápita de energía \\
\hline 18 & Porcentaje de negocios con programas de conservación de energía \\
\hline 19 & Porcentaje de uso de energías renovables \\
\hline 20 & Total de $\mathrm{CO}_{2}$ producido por consumo energético \\
\hline
\end{tabular}

Fuente: elaboración propia con base en OMT (2005) y Medrano y Lardiés (2014).

La tabla 2 presenta ocho indicadores ambientales que se utilizan como línea base para la construcción del IATS, por cumplir con los cuatro criterios sugeridos por Medrano y Lardiés (2014).

Tabla 2. Indicadores por dimensión que cumplen todos los criterios

\begin{tabular}{lc}
\hline \multicolumn{2}{c}{ Planificación del territorio } \\
\hline 1 & Extensión de áreas protegidas (clasificadas por categorías de uso) \\
\hline 2 & Existencia de algún proceso de planificación territorial que incluya el turismo \\
\hline 3 & Residuos sólidos \\
\hline 4 & Volumen de residuos producidos en el destino \\
\hline & Paisibilidad del cielo nocturno \\
\hline 5 & Masificación \\
\hline 6 & Densidad de turistas \\
\hline 7 & Densidad de vehículos utilizados en el sitio \\
\hline 8 & Indicador del grado de presión (capacidad de carga) \\
\hline
\end{tabular}

Fuente: elaboración propia con base en OMT (2005) y Medrano y Lardiés (2014).

Para cada uno de los indicadores presentados en la tabla 2, se elaboró la correspondiente ficha técnica, en la que se describe: la importancia y utilidad del indicador; el método de cálculo; la escala de valoración; la pertinencia, viabilidad y limitaciones de la recogida de datos para el indicador; y la disponibilidad de la información.

Cada indicador se analiza por separado y se procede, con base en la información disponible para el desierto de la Tatacoa, a calcular su valor. Una vez obtenido el resultado numérico, se procede a clasificarlo de acuerdo con la escala de valoración Likert, y se utilizan los ítems con sus correspondientes puntajes: muy bajo (1), bajo (2), moderado (3), bueno (4) y muy bueno (5). Para finalizar, se hace un promedio 
aritmético de las ocho puntuaciones para obtener el IATS. Este índice sintético propio es categórico ordinal de nivel y toma valores desde muy bajo hasta muy bueno, como lo muestra la tabla 3.

Tabla 3. Índice ambiental para el turismo sostenible (IATS)

\begin{tabular}{cccccc}
\hline $\begin{array}{c}\text { Índice ambiental para el } \\
\text { turismo sostenible (IATS) }\end{array}$ & Muy bajo & Bajo & Moderado & Bueno & Muy bueno \\
\hline Valor del IATS & $\begin{array}{c}\text { Entre 0 y } \\
1,5\end{array}$ & $\begin{array}{c}\text { Entre 1,51 } \\
\text { y 2,5 }\end{array}$ & $\begin{array}{c}\text { Entre 2,51 } \\
\text { y 3,5 }\end{array}$ & $\begin{array}{c}\text { Entre 3,51 } \\
\text { y 4,5 }\end{array}$ & Mayor a 4,5 \\
\hline
\end{tabular}

Fuente: elaboración propia.

El IATS sirve como línea base para 2019 y permite valorar, de acuerdo a la información disponible, el estado del turismo sostenible desde el punto de vista ambiental para el desierto de la Tatacoa. En él se tiene en cuenta la especificidad del territorio, por lo que su análisis a través del tiempo, permitirá precisar las potencialidades y las vulnerabilidades sostenibles del sitio turístico.

\section{RESULTADOS}

El análisis de la propuesta presentada por la OMT (2005) sobre indicadores para el desarrollo sostenible de sitios turísticos, ha permitido realizar un listado de indicadores para el desierto de la Tatacoa en el ámbito ambiental reducido (aplicando las consideraciones mencionadas en la metodología) (tabla 2), para obtener el IATS del 2019. Los indicadores seleccionados están basados en datos estadísticos o con un tratamiento de datos simple, de los cuales el 50 \% son proporciones o porcentajes y tres están representados en cifras brutas. Además, se incluye la existencia de algún proceso de planificación territorial que incluya el turismo, como un indicador normativo que se mide de manera cualitativa. A continuación, se presenta el análisis por dimensiones de los indicadores seleccionados.

\subsection{Planificación del territorio}

La ordenación del territorio está dirigida a dar respuesta a los retos que plantea la utilización del espacio físico. En este sentido, con base en la zonificación prevista en el Decreto 1076 de 2015 del Ministerio de Ambiente y Desarrollo Sostenible y dentro del área propuesta como Distrito Regional de Manejo Integrado (DRMI) la Tatacoa, se han clasificado las siguientes categorías de uso del territorio (ver gráfico 1): 22,1 \% zona de preservación $(\mathrm{ZPR})^{2} ;$ 49,9 \% zona de restauración para la preservación

2 "Corresponde al espacio del DRMI donde el manejo estará prioritariamente dirigido a evitar la alteración, degradación o transformación por actividad humana de los valores naturales" (CAM, 2015, p. 71). 
$\left(\right.$ ZREP) 3 ; 8,6 \% zona de restauración para el uso sostenible (ZRUS) ${ }^{4} ; 10,3$ \% zona de uso sostenible (ZUS) ${ }^{5}$; 5,5 \% zona de uso público para la recreación (ZUPR) y 3,6 \% zona de uso público alta densidad de uso (Zupad) ${ }^{7}$.

\section{Gráfico 1. Zonificación del Distrito Regional de Manejo Integrado (DRMI) la Tatacoa}

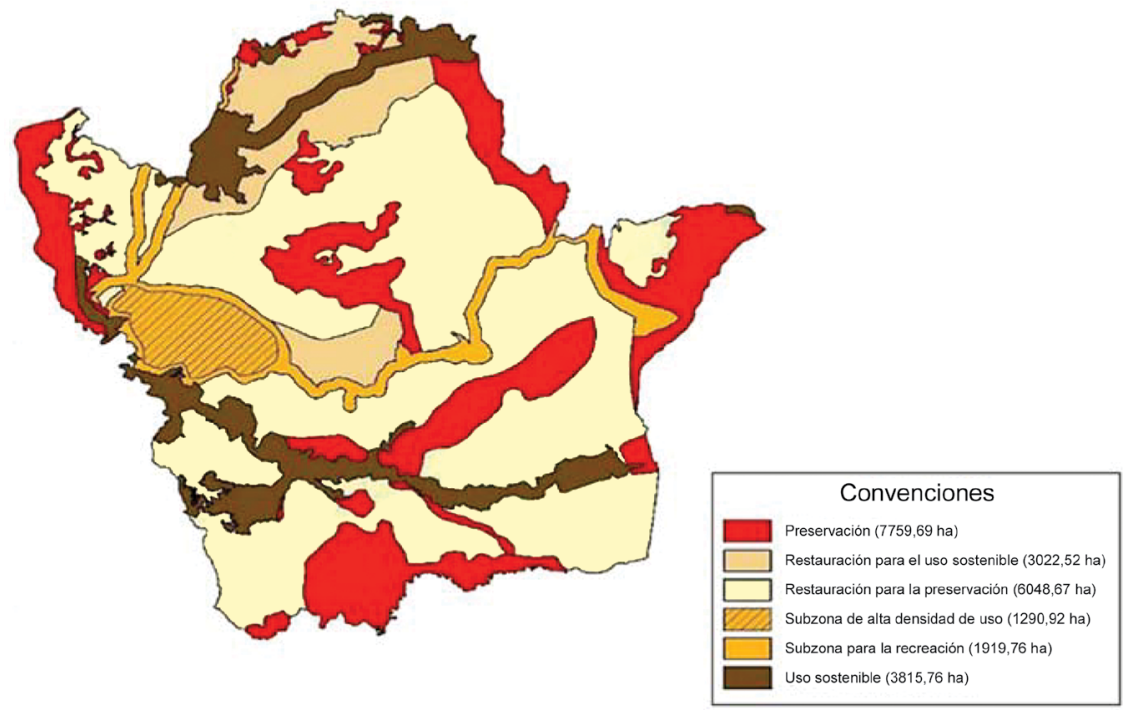

Fuente: CAM $(2015$, p. 70).

Con base en esta información y en la correspondiente ficha técnica de la tabla 4, se calcula el indicador extensión de áreas protegidas, sin incluir la ZUPR y la Zupad; así se obtiene una ponderación de 89,51 \%, es decir, una escala de valoración de muy bueno.

3 "Corresponde aquellos sectores donde se hace necesario el restablecimiento de las condiciones originales de los ecosistemas para recuperar los procesos ecológicos a ellos inherentes" (CAM, 2015, p. 73).

4 "Está conformada por aquellos sitios, desprovisto de cobertura vegetal natural, y sin especial importancia para la preservación, que habiendo sido utilizados en actividades de producción agrícola o pecuaria, han perdido la capacidad productiva de sus suelo, y por ende deben ser objeto de procesos de recuperación para su posterior utilización económica bajo prácticas de manejo que sean compatibles con los objetivos de conservación del DRMI" (CAM, 2015, p. 75).

5 "Es un espacio donde sus condiciones biofísicas y socioeconómicas permiten el desarrollo de actividades productivas de forma sostenible, sin que ellas comprometan los objetivos de conservación" (CAM, 2015, p. 76).

6 "La Zona de Uso Público (ZUP) es un espacio definido para adelantar las actividades con el fin de alcanzar los objetivos de conservación a través de la educación, la recreación, el ecoturismo y el desarrollo de infraestructura de apoyo a la investigación. Contiene además las siguientes subzonas. Subzona para la recreación Es aquella porción, en la que se permite el acceso a los visitantes a través del desarrollo de infraestructura mínima tal como senderos o miradores" (CAM, 2015, p. 78).

7 "Es aquella porción, en la que se permite el desarrollo controlado de infraestructura mínima para el acojo de los visitantes y el desarrollo de facilidades de interpretación" (CAM, 2015, p. 79). 
Tabla 4. Fichas técnicas de los indicadores para la planificación del territorio

\begin{tabular}{|c|c|c|}
\hline Dimensión/indicador/ID & $\begin{array}{c}\text { Planificación del } \\
\text { territorio }\end{array}$ & $\begin{array}{l}\text { Extensión de áreas protegidas } \\
\text { (clasificadas por categorías de } \\
\text { uso) }\end{array}$ \\
\hline $\begin{array}{l}\text { Importancia y utilidad del } \\
\text { indicador }\end{array}$ & $\begin{array}{r}\text { El área protegida pued } \\
\text { potencial de especie } \\
\text { la normatividad, hay } \\
\text { Colomb }\end{array}$ & $\begin{array}{l}\text { una medida importante de la protección } \\
\text { ve (atractivo turístico). Dependiendo de } \\
\text { os niveles diferentes de protección. En } \\
\text { e el Decreto } 1076 \text { de } 2015 \text {. }\end{array}$ \\
\hline
\end{tabular}

Método del cálculo

El indicador corresponde al número total de hectáreas según categorías de uso (ZPR, ZREP, ZRUS, ZUS), expresado como porcentaje del número total de hectáreas del DRMI la Tatacoa.

Este indicador se mide en porcentaje. El rango de variabilidad per-

Escala de valoración misible va desde muy bajo para calificaciones menores a $15 \%$, bajo entre el 15,1 al $25 \%$, moderado de $25,1 \%$ a $50 \%$, bueno de $50,1 \%$ a $75 \%$ hasta muy bueno con calificaciones mayores a $75 \%$.

La zonificación de áreas protegidas define usos específicos y niveles de protección, a menudo con zonas centrales de conservación,

Pertinencia, viabilidad y limitaciones de la recogida

de datos para este indicador zonas de acceso turístico limitado y zonas de amortiguación con un uso más intensivo de las instalaciones para visitantes. Si los efectos son muy graves, quizá se limite o deniegue el acceso al turismo. Para el cálculo de este indicador, en la investigación, no se tuvieron en cuenta las zonas de uso público (ZUPR, ZUPAD), por considerarse de alta vulnerabilidad ambiental.

\begin{tabular}{ccc}
$\begin{array}{c}\text { Disponibilidad de la } \\
\text { información }\end{array}$ & $\begin{array}{c}\text { CAM (2015). Plan de manejo ambiental Distrito Regional de Manejo Integrado } \\
\text { (DRMI) la Tatacoa. Neiva: CAM. }\end{array}$ \\
\hline Dimensión/indicador/ID & $\begin{array}{c}\text { Planificación del } \\
\text { territorio }\end{array}$ & $\begin{array}{c}\text { Existencia de algún proceso } \\
\text { de planificación territorial que } \\
\text { incluya el turismo }\end{array}$ \\
\hline $\begin{array}{c}\text { Importancia y utilidad del } \\
\text { indicador }\end{array}$ & $\begin{array}{c}\text { Sirve para demostrar a las administraciones correspondientes la } \\
\text { conveniencia de poner en marcha algún proceso de planificación en } \\
\text { caso de que no exista. Este indicador puede utilizarse en el futuro } \\
\text { para constatar el porcentaje de destinos o sitios que han puesto en } \\
\text { marcha planes o controles sobre el desarrollo. }\end{array}$ \\
\hline Método del cálculo & $\begin{array}{c}\text { Este indicador es categórico ordinal de nivel, toma valores desde } \\
\text { muy bajo hasta muy bueno. }\end{array}$ \\
\hline Escala de valoración & $\begin{array}{c}\text { Este indicador es cualitativo. El rango de variabilidad permisible va } \\
\text { desde muy bajo, si no existe un proceso de planificación territorial } \\
\text { que incluya el turismo; bajo, si existe un proceso de planificación } \\
\text { territorial que incluya el turismo, pero aún no es oficial; moderado, si } \\
\text { existe un proceso de planificación territorial que incluya el turismo, y } \\
\text { está aprobado el plan en forma oficial; bueno, si existen criterios es- } \\
\text { pecíficos para el control del desarrollo turístico descrito en el plan; } \\
\text { muy bueno, si se realiza la inspección, control y correspondientes } \\
\text { sanciones por incumplimiento del plan. }\end{array}$ \\
\hline
\end{tabular}




\begin{tabular}{cc}
\hline $\begin{array}{c}\text { Pertinencia, viabilidad y } \\
\text { limitaciones de la recogida } \\
\text { de datos para este } \\
\text { indicador }\end{array}$ & $\begin{array}{c}\text { Este indicador es útil para efectuar la comparación a lo largo del } \\
\text { tiempo y demostrar los resultados de la aplicación de los procesos } \\
\text { de planificación en el destino turístico. }\end{array}$ \\
\hline $\begin{array}{c}\text { Disponibilidad de la } \\
\text { información }\end{array}$ & Alcaldía de Villavieja. \\
\hline
\end{tabular}

Fuente: elaboración propia.

Asimismo, se identifica utilizando la ficha técnica de la tabla 4, que existe un proceso de planificación territorial que incluye el turismo, pero aún no es oficial por lo que se valoró como bajo. En la web (consultada en febrero de 2020) se encuentra un documento preliminar del Plan de Ordenamiento Territorial (POT), en el cual se incluye el sector turismo. Junto con la aprobación del POT se hace necesario adelantar un plan de desarrollo turístico sostenible para el desierto de la Tatacoa que permita mejorar su competitividad.

\subsection{Residuos sólidos}

Con respecto a la generación de residuos sólidos en el desierto de la Tatacoa, Empresas Públicas de Villavieja (EPV, 2019), informa que realiza la recolección, transporte y disposición final de los residuos inorgánicos. Adicionalmente, EPV SAS ESP., está ubicando contenedores para manejo de residuos peligrosos como pilas, baterías de teléfonos celulares, controles y demás residuos electrónicos, lo que denota una política local que ha incentivado el manejo adecuado de los residuos y unos ciudadanos conscientes de la separación en fuente que, de acuerdo con Sánchez, Cruz y Maldonado (2019), es indispensable para la consecución del desarrollo sostenible.

En el gráfico 2 se observa el comportamiento mensual entre el número de habitantes permanentes y temporales con respecto a la generación de residuos sólidos por kilo al mes. Los habitantes permanentes corresponden a los residentes, mientras que los temporales son los turistas. En los meses de diciembre de $2018 \mathrm{y}$ marzo de 2019, la generación alcanza sus topes más altos (3.200 y 2.700 kilos/mes, respectivamente) por ser la época de temporada alta para este destino turístico.

Con la información del gráfico 2 y con base en la ficha técnica de la tabla 5 , se calcula el indicador de volumen de residuos sólidos; este indicador arroja una escala de valoración de muy bueno en los meses de noviembre de 2018, enero y abril de 2019. Por su parte, en los meses de diciembre de 2018 y marzo de 2019 la escala de valoración fue de muy bajo. En promedio, el desierto de la Tatacoa recibe una puntuación de 0,51 que lo clasifica en una escala moderada. 
Gráfico 2. Generación de residuos sólidos inorgánicos y habitantes en el desierto de la Tatacoa

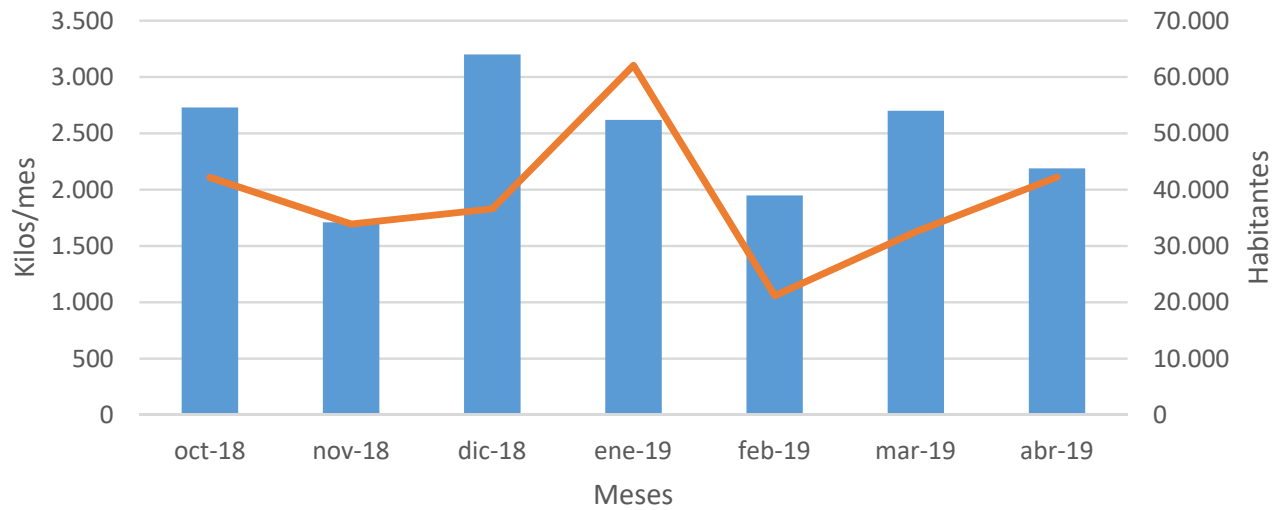

Generación de residuos sólidos $\quad$ Habitantes permanentes y temporales

Fuente: elaboración propia con base en Empresas Públicas de Villavieja (2019); Oficina de Turismo de Villavieja (2019) y DNP (2018).

Tabla 5. Fichas técnicas de los indicadores para los residuos sólidos

\begin{tabular}{|c|c|c|c|}
\hline Dimensión/indicador/ID & Residuos sólidos & $\begin{array}{l}\text { Volumen de residuos } \\
\text { producidos en el destino }\end{array}$ & I3 \\
\hline $\begin{array}{l}\text { Importancia y utilidad del } \\
\text { indicador }\end{array}$ & \multicolumn{3}{|c|}{$\begin{array}{c}\text { El envío de materiales de desecho al relleno sanitario representa } \\
\text { una pérdida de recursos y su reposición incrementa la emisión de } \\
\text { gases de efecto invernadero durante la producción y el transporte. } \\
\text { El indicador resulta útil para ver las tendencias de la producción } \\
\text { de residuos sólidos y hacer un monitoreo en el destino que } \\
\text { permita adoptar las medidas oportunas. }\end{array}$} \\
\hline Método del cálculo & \multicolumn{3}{|c|}{$\begin{array}{l}\text { Se toma el volumen de residuos producidos en el destino (kilos) } \\
\text { al mes entre la población residente en forma permanente y } \\
\text { temporal (turismo) al mes. El promedio aritmético de la serie de } \\
\text { datos se normaliza basado en la unidad. }\end{array}$} \\
\hline Escala de & \multicolumn{3}{|c|}{$\begin{array}{c}\text { Este indicador se mide entre } 0 \text { y } 1 \text {. El rango de variabilidad va } \\
\text { desde muy bajo para valores mayores a } 0,9 \text {; bajo entre } 0,71 \text { y } 0,9 \text {; } \\
\text { moderado de } 0,51 \text { a } 0,7 \text {; bueno de } 0,31 \text { a } 0,5 \text {; hasta muy bueno } \\
\text { con calificaciones menores a } 0,3 \text {. }\end{array}$} \\
\hline $\begin{array}{l}\text { Pertinencia, viabilidad y } \\
\text { limitaciones de la recogida } \\
\text { de datos para este indicador }\end{array}$ & \multicolumn{3}{|c|}{$\begin{array}{l}\text { Este indicador puede analizarse de dos formas: a lo largo del } \\
\text { tiempo en el mismo lugar de destino o en comparación con los } \\
\text { datos de otros lugares de destino. En el desierto de la Tatacoa, las } \\
\text { fuentes de información permiten su obtención mes a mes. }\end{array}$} \\
\hline $\begin{array}{l}\text { Disponibilidad de la } \\
\text { información }\end{array}$ & \multicolumn{3}{|c|}{$\begin{array}{c}\text { Empresas Públicas de Villavieja, Coordinación de Turismo Local de } \\
\text { Villavieja y Departamento Nacional de Planeación. }\end{array}$} \\
\hline
\end{tabular}




\begin{tabular}{|c|c|c|}
\hline Dimensión/indicador/ID & Residuos sólidos & $\begin{array}{l}\text { Número de establecimientos } \\
\text { turísticos que reciclan sus } \\
\text { propios residuos }\end{array}$ \\
\hline $\begin{array}{l}\text { Importancia y utilidad del } \\
\text { indicador }\end{array}$ & \multicolumn{2}{|c|}{$\begin{array}{l}\text { La base del reciclaje está en separar los diferentes tipos de } \\
\text { residuos, algo que es conveniente hacer en origen. Por tanto, es } \\
\text { importante verificar si los establecimientos turísticos efectúan } \\
\text { una recogida diferenciada de basuras. Este indicador es útil para } \\
\text { examinar tendencias de reciclaje en el lugar de destino y controlar } \\
\text { los resultados de las actividades realizadas. }\end{array}$} \\
\hline Mét & \multicolumn{2}{|c|}{$\begin{array}{l}\text { Número total de establecimientos turísticos que reciclan, expresa- } \\
\text { do como el porcentaje del total de establecimientos turísticos en } \\
\text { el destino. }\end{array}$} \\
\hline Escala & \multicolumn{2}{|c|}{$\begin{array}{l}\text { Este indicador se mide en porcentaje. El rango de variabilidad } \\
\text { permisible va desde muy bajo para calificaciones menores a } 15 \% \text {; } \\
\text { bajo entre el } 15,1 \% \text { al } 25 \% \text {; moderado de } 25,1 \% \text { a } 50 \% \text {; bueno de } \\
50,1 \% \text { a } 75 \% \text {; hasta muy bueno con calificaciones mayores a } 75 \% \text {. }\end{array}$} \\
\hline $\begin{array}{l}\text { Pertinencia, viabilidad y } \\
\text { limitaciones de la recogida } \\
\text { de datos para este indicador }\end{array}$ & \multicolumn{2}{|c|}{$\begin{array}{l}\text { Este indicador puede analizarse de dos formas: a lo largo del } \\
\text { tiempo en el mismo lugar de destino o en comparación con los } \\
\text { datos de otros lugares de destino. En el desierto de la Tatacoa, } \\
\text { los datos se obtienen de las fuentes de información oficiales } \\
\text { a través de entrevistas, dado que no se dispone de informes } \\
\text { periódicos al respecto. }\end{array}$} \\
\hline $\begin{array}{l}\text { Disponibilidad de la } \\
\text { información }\end{array}$ & \multicolumn{2}{|c|}{$\begin{array}{l}\text { Empresas Públicas de Villavieja y Coordinación de Turismo Local } \\
\text { de Villavieja. }\end{array}$} \\
\hline
\end{tabular}

Fuente: elaboración propia.

Con respecto al indicador sobre el número de establecimientos turísticos que reciclan sus propios residuos (tabla 5), el puntaje es del $100 \%$ (muy bueno), dado que, de acuerdo con Hernández (2019), el $100 \%$ de los establecimientos de turismo existentes en el destino (veintiséis en total) hacen separación en fuente con fines de reciclaje. Asimismo, Empresas Pública de Villavieja (EPV, 2019) afirma que cada establecimiento se encarga de la comercialización del material reciclado. Se requiere de campañas agresivas dirigidas a los habitantes temporales para que apliquen hábitos adecuados con la generación de sus residuos sólidos en el sitio turístico.

\subsection{Paisaje}

Para esta dimensión se toma el indicador visibilidad del cielo nocturno porque la iluminación artificial de un territorio turístico debe estar restringida y controlada para evitar interferencias en el ciclo de vida nocturno de las especies silvestres. También es importante evitar la propagación de luz en el horizonte para permitir una clara visibilidad de las estrellas por la noche. Este indicador permite hacer seguimiento 
para proteger el cielo oscuro y poder desarrollar el turismo de estrellas, dado que el cielo nocturno estrellado es uno de los recursos naturales del desierto de la Tatacoa que desde hace varios años se viene aprovechando a nivel turístico.

En el 2019 el desierto de la Tatacoa obtuvo la certificación Starlight, la cual acredita la calidad de los cielos con parámetros como transparencia y brillo del cielo. Dicha certificación hace referencia a territorios que son excelentes para la observación de las estrellas. La certificación favorece el turismo sostenible en el desierto porque permite que las condiciones de luminosidad sean monitoreadas y controladas a través del tiempo. Este sistema de certificación está respaldado por la Unesco, la OMT y la Unión Astronómica Internacional (UAI).

Al tener en cuenta los criterios establecidos para la certificación, el desierto de la Tatacoa obtiene una puntuación de 84 \% (veintiún puntos sobre veinticinco), lo que lo ubica en una escala de valoración de muy bueno. Sin embargo, aspectos como: la falta de un plan en materia de prevención de la contaminación lumínica respecto a las instalaciones de alumbrado exterior de las municipalidades próximas; la inexistencia de una normativa que cumpla con criterios sostenibles respecto al alumbrado exterior; la insuficiente renovación del alumbrado exterior actual y la desproporción entre el número de luminarias y la necesidad real de iluminación en la zona, deben ser tenidos en cuenta para lograr futuras renovaciones de la certificación.

\subsection{Masificación}

En esta dimensión se tienen en cuenta tres indicadores: densidad de turistas (I6), densidad de vehículos utilizados en el sitio (I7) y grado de presión (capacidad de carga) (I8), cuyas fichas técnicas se encuentran en la tabla 6.

Tabla 6. Fichas técnicas de los indicadores para la masificación

\begin{tabular}{ccc}
\hline Dimensión/indicador/ID & Masificación & Densidad de turistas \\
\hline $\begin{array}{c}\text { Importancia y utilidad del } \\
\text { indicador }\end{array}$ & $\begin{array}{c}\text { Es un indicador básico para la administración de cualquier destino } \\
\text { turístico. Se considera que la densidad de uso turístico está relacio- } \\
\text { nada y, en ocasiones, ayuda a predecir el nivel de presión que sufren } \\
\text { los bienes naturales y la infraestructura, así como los niveles de } \\
\text { gestión y mitigación necesarios y otros aspectos de la sostenibilidad } \\
\text { a largo plazo. }\end{array}$ \\
\hline Método del cálculo & $\begin{array}{c}\text { Número promedio de turistas diarios en el sitio por kilómetro } \\
\text { cuadrado. El promedio aritmético de la serie de datos se normaliza } \\
\text { basado en la unidad. }\end{array}$ \\
\hline
\end{tabular}




\begin{tabular}{|c|c|}
\hline Escala de valoración & $\begin{array}{c}\text { Este indicador se mide entre } 0 \text { y } 1 \text {. El rango de variabilidad va } \\
\text { desde muy bajo para valores mayores a } 0,9 \text {; bajo entre } 0,71 \text { y } 0,9 ; \\
\text { moderado de } 0,51 \text { a } 0,7 \text {; bueno de } 0,31 \text { a } 0,5 \text {; hasta muy bueno con } \\
\text { calificaciones menores a } 0,3 \text {. }\end{array}$ \\
\hline $\begin{array}{l}\text { Pertinencia, viabilidad y } \\
\text { limitaciones de la reco- } \\
\text { gida de datos para este } \\
\quad \text { indicador }\end{array}$ & $\begin{array}{c}\text { La intensidad de uso turístico se ha calculado en diferentes estudios } \\
\text { que utilizan el indicador de número de turistas por metro o kiló- } \\
\text { metro cuadrado. Este indicador puede emplearse para demostrar } \\
\text { la necesidad de nuevas infraestructuras y analizar problemas de } \\
\text { turismo masivo. }\end{array}$ \\
\hline $\begin{array}{l}\text { Disponibilidad de la } \\
\text { información }\end{array}$ & $\begin{array}{l}\text { Coordinación de Turismo Local de Villavieja. CAM (2015) Plan de manejo } \\
\text { ambiental - Distrito Regional de Manejo Integrado- DRMI la Tatacoa. }\end{array}$ \\
\hline Dimensión/indicador/ID & $\begin{array}{l}\text { Densidad de vehículos } \\
\text { utilizados en el sitio }\end{array}$ \\
\hline $\begin{array}{l}\text { Importancia y utilidad del } \\
\quad \text { indicador }\end{array}$ & $\begin{array}{l}\text { El principal medio de llegada de turistas al desierto de la Tatacoa es } \\
\text { el vehículo. La densidad de automóviles puede ser más importante } \\
\text { en cuanto la masificación. }\end{array}$ \\
\hline Método del cálculo & $\begin{array}{c}\text { Número promedio de vehículos diarios utilizados en el sitio por } \\
\text { kilómetro cuadrado. El promedio aritmético de la serie de datos se } \\
\text { normaliza basado en la unidad. }\end{array}$ \\
\hline Escala de valoración & $\begin{array}{c}\text { Este indicador se mide entre } 0 \text { y } 1 \text {. El rango de variabilidad va } \\
\text { desde muy bajo para valores mayores a } 0,9 \text {; bajo entre } 0,71 \text { y } 0,9 ; \\
\text { moderado de } 0,51 \text { a } 0,7 \text {; bueno de } 0,31 \text { a } 0,5 \text {; hasta muy bueno con } \\
\text { calificaciones menores a } 0,3 \text {. }\end{array}$ \\
\hline $\begin{array}{l}\text { Pertinencia, viabilidad y } \\
\text { limitaciones de la reco- } \\
\text { gida de datos para este } \\
\quad \text { indicador }\end{array}$ & $\begin{array}{l}\text { El uso de la densidad de vehículos por kilómetro cuadrado es } \\
\text { suficiente para analizar la masificación turística del sitio. Igualmente, } \\
\text { los cambios de la densidad de vehículos a lo largo del tiempo } \\
\text { proporcionan información de utilidad a los tomadores de decisiones. }\end{array}$ \\
\hline $\begin{array}{l}\text { Disponibilidad de la } \\
\text { información }\end{array}$ & $\begin{array}{l}\text { Coordinación de Turismo Local de Villavieja. CAM (2015). Plan de } \\
\text { manejo ambiental - Distrito Regional de Manejo Integrado- DRMI } \\
\text { la Tatacoa. }\end{array}$ \\
\hline Dimensión/indicador/ID & $\begin{array}{l}\text { Indicador del grado de } \\
\text { presión (capacidad de carga) }\end{array}$ \\
\hline $\begin{array}{l}\text { Importancia y utilidad del } \\
\text { indicador }\end{array}$ & $\begin{array}{c}\text { El desarrollo turístico sostenible va unido al concepto de ca- } \\
\text { pacidad de carga. Dicho concepto significa el nivel máximo de } \\
\text { visitantes que un área determinada puede soportar con el menor } \\
\text { impacto ambiental. }\end{array}$ \\
\hline Método del cálculo & $\begin{array}{c}\text { El indicador corresponde al número total de turistas por día que } \\
\text { excede la capacidad de carga turística (CCT) de los senderos } \\
\text { Xilópalos, Los Hoyos y Cusco, junto con el Observatorio } \\
\text { Astronómico del desierto de la Tatacoa. }\end{array}$ \\
\hline
\end{tabular}


Este indicador se mide en porcentaje. El rango de variabilidad permisible va desde muy bajo para calificaciones mayores a $25 \%$; bajo entre el $0,1 \%$ al $25 \%$, moderado de $0 \%$; bueno para valores

Escala de valoración mayores al $-25 \%$ y menores al $0 \%$; hasta muy bueno con calificaciones menores al $-25,1 \%$. La escala de valoración de bueno y muy bueno indican que el número de turistas por día se encuentra por debajo de la capacidad de carga.

Pertinencia, viabilidad y limitaciones de la recogida de datos para este indicador

Disponibilidad de la información
Este indicador es una herramienta de planificación que sustenta y requiere de decisiones de manejo. A su vez, es relativa y dinámica

porque depende de variables que puede cambiar según las circunstancias lo que obliga a revisiones periódicas como parte de un proceso secuencial de planificación.

CAM (2014). Ordenamiento ecoturístico, en el cual se incluya determinar la capacidad de carga real, física, de manejo y efectiva del PNR la Tatacoa y su área de influencia. Neiva: Corporación Autónoma del Alto Magdalena y Coordinación de Turismo Local de Villavieja.

Fuente: elaboración propia.

Con respecto a la densidad de turistas y de vehículos en el gráfico 3 se observa que en los meses de temporada alta (enero, abril, julio) y de actividades programadas como el Festival de la Tatacoa en octubre, aumenta la densidad. En este último mes el número promedio de turistas diarios por $\mathrm{km}^{2}$ es de 199 y de vehículos 27. Los meses de más baja densidad registrada son febrero (treinta y seis turistas y nueve vehículos) y septiembre de 2019.

\section{Gráfico 3. Densidad de turistas y de vehículos en el desierto de la Tatacoa}

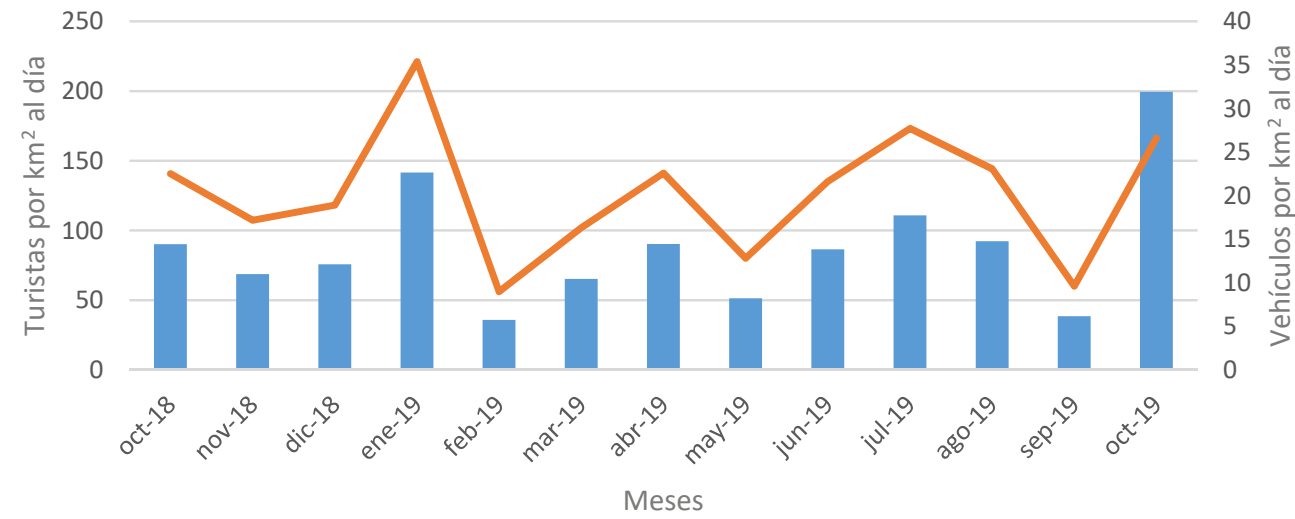

Densidad de turistas Densidad de vehículos

Fuente: elaboración propia con base en Hernández (2019) y CAM (2015). 
Al revisar los valores de cada indicador (I6 e I7) con respecto a la escala de valoración, el desierto de la Tatacoa se encuentra en una puntuación buena. Esto se debe a que los 12,9 kilómetros cuadrados de la subzona de uso público para la recreación asumen dispersión de la actividad turística. Sin embargo, de acuerdo con Giraldo y Ramírez (2016), en el territorio se desarrolla el turismo en lugares puntuales como los senderos y el observatorio astronómico, y esto genera un intenso impacto medioambiental y mayor riesgo de masificación, saturación e incomodidad del turista, por lo que se hace necesario analizar la capacidad de carga turística.

De acuerdo con la CAM (2014), la capacidad de carga turística ${ }^{8}$ calculada para el desierto de la Tatacoa es en total de doscientos veintinueve turistas máximo que pueden visitar por día los cuatro atractivos turísticos: senderos Xilópalos, Los Hoyos, Cusco y el Observatorio Astronómico. En este sentido, al medir el indicador grado de presión (capacidad de carga) (I8), expresado en la tabla 6, se obtienen valores que superan la capacidad de carga del ecosistema y deja al desierto en una escala de valoración muy malo. Así, al tomar como criterio que un turista visita mínimo dos atractivos turísticos al día, el grado de presión promedio es de $148 \%$.

Por lo anterior, según Hernández (2019), la CAM está estudiando la posibilidad de abrir quince nuevos senderos ecoturísticos y está reorganizando cinco senderos, entre los que se encuentran: sendero Hoyos, sendero Cusco, sendero Cardón, sendero Xilópalos y sendero Piedra Pintada. De esta manera, ha aumentado la capacidad de carga del desierto de la Tatacoa al doble, al elaborar políticas de dispersión que, de acuerdo con Vera (2013), hacen que los espacios turísticos posean

un crecimiento de la oferta y la demanda de carácter extensivo, suponiendo un impacto medioambiental más disperso sobre el turismo y de menor intensidad. En estos espacios existe un riesgo de que al final todo quede transformado por el turismo. (p. 210)

Con base en los ocho indicadores antes analizados, en la tabla 7 se resume su valoración y se presenta el cálculo del IATS en el desierto de la Tatacoa. Este índice sintético presenta una valoración ambiental buena para el destino turístico, y se destaca la visibilidad del cielo nocturno con la certificación Starlight y el número de establecimientos que reciclan sus residuos. El IATS, como línea base ambiental 2019, aporta a la descripción de la influencia de la actividad turística con el fin de evaluar a través del tiempo los efectos que pudieren generarse o presentarse sobre los elementos del medio ambiente.

La capacidad de carga turística está formada por tres componentes: capacidad de carga física (CCF), capacidad de carga real (CCR) y capacidad de carga de manejo (CCM). 


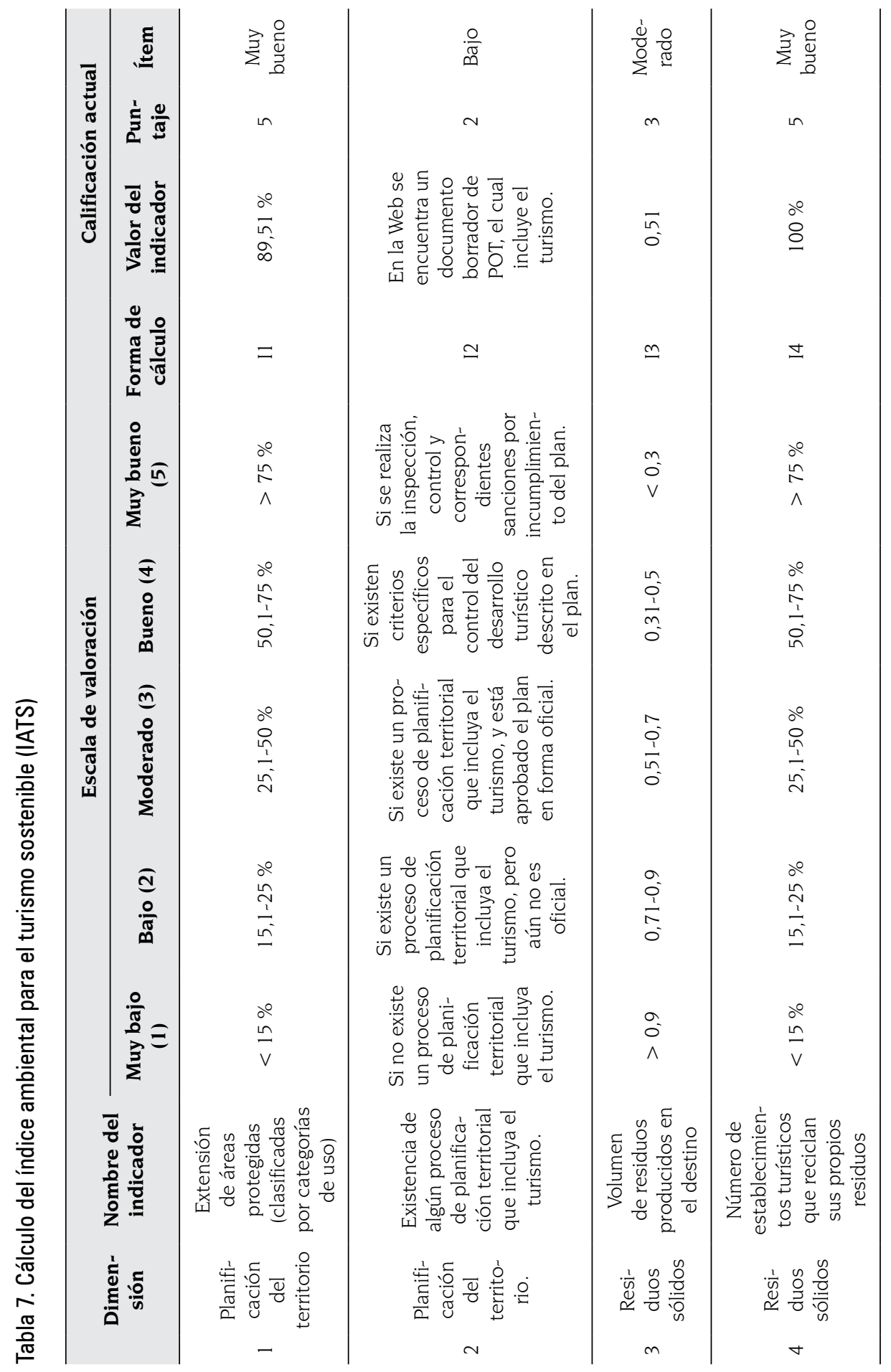




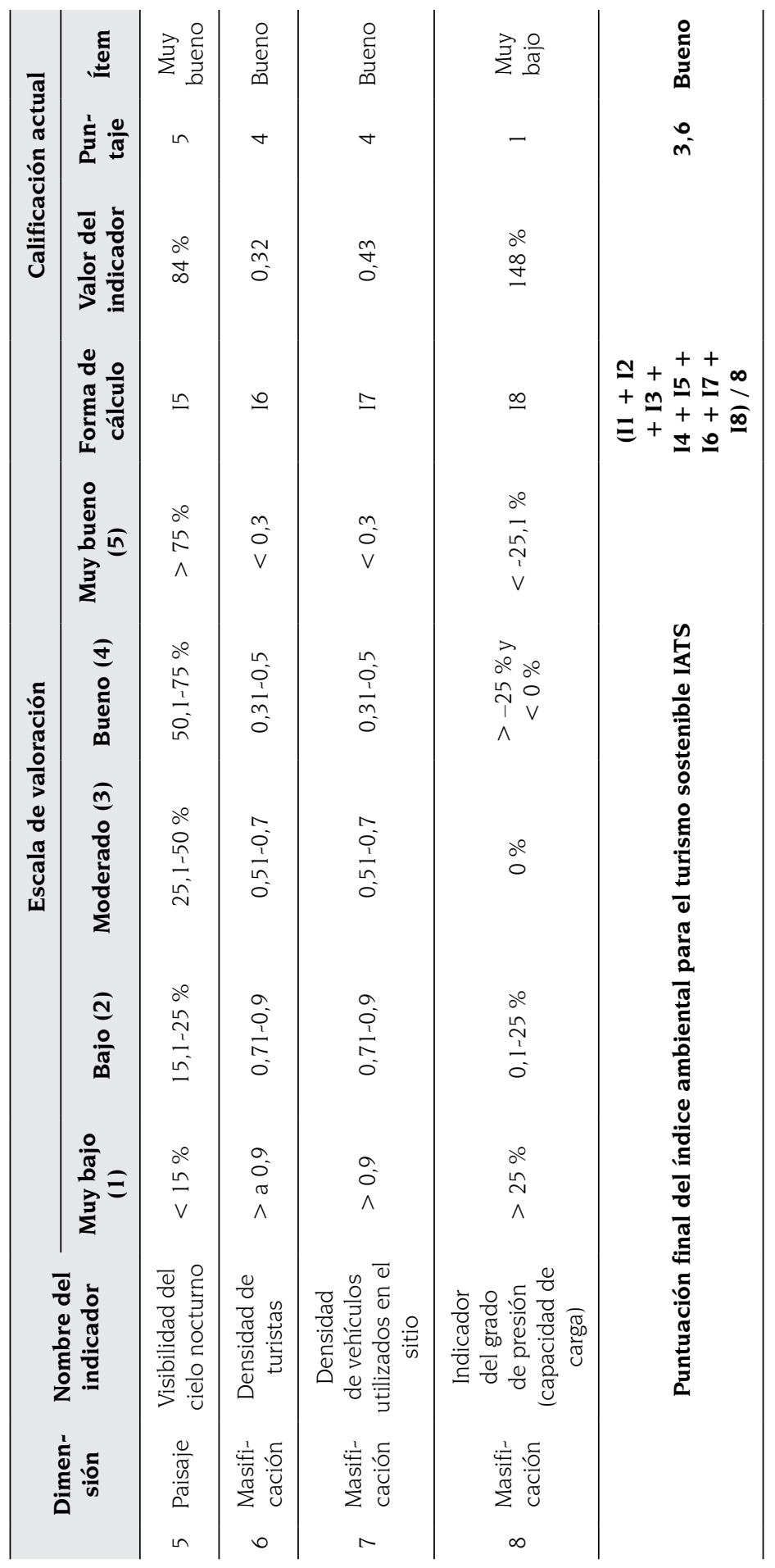




\section{CONCLUSIONES}

El trabajo logra diseñar una propuesta de indicadores ambientales para un turismo sostenible en el desierto de la Tatacoa, a través de la cual ha sido posible cuantificar y plasmar parte de los efectos causados por esta actividad en el ambiente.

Mediante la revisión de la literatura se corrobora que no existe una única forma de cuantificar el efecto ambiental del turismo, dado que los criterios de selección de los indicadores están supeditados a subjetividad. Asimismo, se observa la falta de datos que permitan aplicar los indicadores. Cuando estos existen, su fiabilidad es escasa debido a una recopilación no sistémica o un uso inapropiado de las fuentes, lo que dificulta y limita los alcances de la investigación.

Por lo anterior, es importante que las entidades encargadas trabajen en coordinación con el sector privado para mejorar el nivel de accesibilidad de información y la elaboración de estadísticas. De todas formas, la valoración e interpretación de los indicadores arroja información útil para la toma de decisiones en lo que respecta a la planificación y gestión turística, de manera que se pueden implementar acciones para mitigar los efectos ambientales y generar un verdadero turismo sostenible.

En relación con la metodología propuesta, cabe mencionar que a todos los indicadores se les dio la misma importancia en el momento de construir el índice sintético IATS. Esto se hizo con el fin de avanzar en un mayor entendimiento de los efectos ambientales del turismo en el desierto de la Tatacoa y ofrecer una herramienta que permita determinar las tendencias y dinámicas de la actividad turística, evaluar los cambios y transformaciones y ofrecer unas recomendaciones a los agentes responsables de la planificación y gestión del destino.

Esta propuesta es un punto de partida para analizar un fenómeno complejo de una manera simple. Es necesario que los veinte indicadores ambientales que no cumplieron con los criterios establecidos, puedan contar en el futuro con información para su correspondiente consideración. Se recomienda, además, que los mismos sean actualizados con regularidad para poder incluir otras dimensiones como calidad del aire, especies, agua, ruido y energía en el índice sintético IATS. Se hace indispensable, entonces, la construcción de una política pública asertiva para su obtención con el fin de valorar y medir de forma más precisa e integral el desarrollo de la actividad turística en el desierto de la Tatacoa.

Finalmente, se invita a los tomadores de decisiones a elaborar el plan de desarrollo turístico sostenible para el desierto de la Tatacoa, en el que se incluyan los aspectos relevantes de las nueve dimensiones mencionadas en esta investigación. 
Especialmente, se resalta la inclusión de temas ambientales, tales como la conservación del paisaje tanto diurno como nocturno; el manejo adecuado de los residuos sólidos por parte de los residentes permanentes y temporales (turistas); la apertura estratégica de los nuevos senderos para evitar exceder la capacidad de carga de los ya existentes y la creación de un sistema estadístico que permita identificar la estructura y evolución del turismo.

\section{BIBLIOGRAFÍA}

CAM - Corporación Autónoma Regional del Alto Magdalena- (2014). Ordenamiento ecoturístico, en el cual se incluya determinar la capacidad de carga real, física, de manejo y efectiva del PNR la Tatacoa y su área de influencia. Neiva: CAM, 171p.

CAM - Corporación Autónoma Regional del Alto Magdalena- (2015). Plan de Manejo Ambiental Distrito Regional de Manejo Integrado (DRMI) la Tatacoa. Neiva: CAM, 117 p.

Cordero, Juana (2017). Propuesta de un sistema de indicadores de sostenibilidad turística para destinos urbanos. En: Revista de Investigación en Turismo, vol. 7, n. ${ }^{\circ}$ 1, p. 41-51.

Car, Adrijana; Miyliyev, Rustam; Sabzaliev, Tae y Paulus, Gernot (2018). Design of a geodatabase for tourism infrastructure in central Asia. En: International Journal of Geoinformatics, vol. $14,{ }^{\circ} 1$, p. $43-52$.

Del Bon, Andrea; Burri, Ezio; Ragni, Pietro; Ferrari, Angelo; Doulati Ardejani, Faramarz y Younesian, Ali (2019). The management of water resources between traditions and sustainability: the Qanats of Shahrood Province (North-Eastern Iran). En: Rendiconti Online Società Geologica Italiana, vol. 47, p. 31-35. DOI: 10.3301/ROL.2019.06

DNP -Departamento Nacional de Planeación- (2018). Ficha de caracterización de Villavieja. https://bit.ly/2NIbWps

Empresas Públicas de Villavieja -EPV- (2019). Presentación de la Gestión y Manejo de Residuos. Villavieja: EPV.

Giraldo, José (2018). Aportaciones a la comprensión de las relaciones entre el turismo sostenible y el territorio sostenible en el desierto de la Tatacoa. Tesis para optar al título de Doctor en Desarrollo Sostenible. Doctorado en Desarrollo Sostenible, Universidad de Manizales, Colombia, 127p.

Giraldo, José y Ramírez, Duván (2016). Territorio, turismo y sostenibilidad en el desierto de la Tatacoa. En: Serna, Ciro (Comp.). Territorio y desarrollo sostenible. Ciudad de México: Ediciones de la U, pp. 79-94.

Hernández, Guillermo (2019). Informe para la Certificación de Turismo Sostenible. Villavieja: Oficina de Turismo Local, Alcaldía de Villavieja. Mimeo.

Khalaf, Ezz; Wahed, Mohamed; Maged, Azeeza y Mokhtar, Hesham (2019). Volcanic Geosites and Their Geoheritage Values Preserved in Monogenetic Neogene Volcanic Field, Bahariya Depression, Western Desert, Egypt: Implication for Climatic Change-Controlling Volcanic Eruption. En: Geoheritage, vol. 11, n. ${ }^{\circ}$ 3, p. 855-873. DOI: 10.1007/s12371-018-0336-6 
Mamani, Wilson (2017). Entender el turismo: glosario básico OMT. https://bit.ly/2CPwOJ6

Medrano, Sara y Lardiés, Raúl (2014). Propuesta de indicadores ambientales para un turismo sostenible en la ciudad de Zaragoza. En: Geographicalia, n. ${ }^{\circ}$ 66, p. 99-132.

OECD - Organisation for Economic Cooperation and Development- (2005). Environment at a Glance: Environmental Indicators. París: OECD, 159p.

Oficina de Turismo de Villavieja (2019). Calendario de ingresos mensuales 2018 y 2019. Villavieja: Oficina de Turismo Local, Alcaldía de Villavieja. Mimeo.

OMT -Organización Mundial del Turismo- (2020). El turismo internacional sigue adelantando a la economía global. https://bit.ly/2NFlTE4

OMT -Organización Mundial del Turismo- (2005). Indicadores de desarrollo sostenible para los destinos turísticos. Madrid: OMT, 545 p.

Sallam, Emad; Abd El-Aal, Ahmed; Fedorov, Yury; Bobrysheva, Olessia y Ruban, Dmitry (2018). Geological heritage as a new kind of natural resource in the Siwa Oasis, Egypt: The first assessment, comparison to the Russian South, and sustainable development issues. En: Journal of African Earth Sciences, vol. 144, p. 151-160. DOI: 10.1016/j.jafrearsci.2018.04.008

Sánchez, María; Cruz, José y Maldonado, Paula (2019). Gestión de residuos sólidos urbanos en América Latina: un análisis desde la perspectiva de la generación. En: Finanzas y Política Económica, vol. 11, n. ${ }^{\circ}$ 2, p. 321-336. DOI: 10.14718/revfinanzpolitecon.2019.11.2.6.

Vera, Fernando (Coord.) (2013). Análisis territorial del turismo y planificación de destinos turísticos. Valencia: Tirant lo Blanch, 485p.

Woyo, Elisha y Amadhila, Elina (2018). Desert tourists experiences in Namibia: A Netnographic Approach. En: African Journal of Hospitality, Tourism and Leisure, vol. 7, n. ${ }^{\circ}$ 3, p. 1-13. 